\title{
Longterm renal outcome of biopsy-proven acute tubular necrosis and acute interstitial nephritis
}

Hyunseo Kim

Korea University Anam Hospital

\section{Sang Kyung}

Korea University Anam Hospital

\section{Shin Young Ahn}

Korea University Guro Hospital

\section{Young Joo Kwon}

Korea University Guro Hospital

\section{Hajeong Lee}

Seoul National University College of Medicine

\section{Jieun Oh}

Hallym University Sacred Heart Hospital

Ho Jun Chin

Seoul National University Bundang Hospital

Ki Joon Lim

Korea University Anam Hospital

Jun Yong Lee

Korea University Anam Hospital

Ji Hyun Yang

Korea University Anam Hospital

\section{Myung-Gyu Kim}

Korea University Anam Hospital

\section{Won Yong Cho}

Korea University Anam Hospital

\section{Sewon Oh ( $\square$ hisy81@hanmail.net)}

Korea University College of Medicine and School of Medicine

\section{Research article}

Keywords: acute interstitial nephritis, acute tubular necrosis, inflammation, fibrosis

Posted Date: April 29th, 2020 
DOI: https://doi.org/10.21203/rs.2.13706/v2

License: (c) (1) This work is licensed under a Creative Commons Attribution 4.0 International License. Read Full License 
The authors have withdrawn this preprint from Research Square 